\title{
Tratamento de paciente com disfunção miofascial craniocervicomandibular ascendente associada à alteração vestibulococlear: relato de caso
}

\author{
Treatment of a patient with ascending craniocervicomandibular disorder associated \\ with vestibulococlear alteration: case report \\ Tratamiento de un paciente con trastorno craniocervicomandibular ascendente asociado \\ con alteración vestibulococlear: reporte de caso \\ Inácio Bruno Romano RIBEIRO \\ Lioney Nobre CABRAL \\ Escola Superior de Ciências do Amazonas - Universidade do Estado do Amazonas, ESA-UEA. Manaus - AM, Brasil. CEP: 69050-030.
}

\section{Resumo}

Dor e disfunção da articulação temporomandibular (DTM) são comuns e resultam de uma ampla variedade de agentes etiológicos, tanto traumáticos quanto não-traumáticos. O conjunto de sinais e sintomas (dor, alteração da função, ruídos articulares) é denominada disfunção temporomandibular (DTM) e, quando abrange também problemas clínicos na região cervical, utiliza-se o termo disfunção craniocervicomandibular (DCCM). Esta condição pode concorrer para as alterações coclear e vestibular, onde a primeira é justificada pela origem embrionária e proximidade anatômica da ATM com a orelha e a segunda, pela disfunção dos músculos da mastigação e cervical (Masseter e Esternocleidomastóideo) que, por serem antigravitacionais, concorrem para a vestibulopatia, e também, otalgias reflexas. Apesar dessas relações existirem sua patogênese não está totalmente esclarecida. Este trabalho tem como objetivo apresentar um caso clínico de diagnóstico e tratamento de paciente com DCCM associado à alteração vestibulococlear.

Descritores: Articulação Temporomandibular; Cervicalgia; Pontos-Gatilho; Transtornos Craniomandibulares; Síndromes da Dor Miofascial.

\section{Abstract}

Temporomandibular joint (TMJ) pain and dysfunction are common and result in a wide variety of etiologic agents, both traumatic and nontraumatic. The set of signs and symptoms (pain, alteration of function, joint noise) is called temporomandibular disorder (TMJ) and, when clinical problems are also used in the cervical region, craniocervicomandibular dysfunction (CCMD) is used. This condition can be performed for cochlear and vestibular alterations, where the first is justified by the embryonic origin and the anatomical proximity of the TMJ to the ear and the second, by the dysfunction of the masticatory and cervical muscles (masseter and sternocleidomastoid) that, by antigravitational reasons, contribute to vestibulopathy and also reflex otalgias. Despite these existing relationships, its pathogenesis is not fully understood. This work aims to present a clinical case of diagnosis and treatment of patient with DCCM associated with vestibulocochlear alteration.

Descriptors: Temporomandibular Joint; Neck Pain; Trigger Points; Craniomandibular Disorders; Myofascial Pain Syndromes.

\section{Resumen}

El dolor y la disfunción de la articulación temporomandibular (TMD) son comunes y resultan en una amplia variedad de agentes etiológicos, tanto traumáticos como no traumáticos. El conjunto de signos y síntomas (dolor, alteración de la función, ruido articular) se llama trastorno temporomandibular (TMD) y, cuando también cubre problemas clínicos en la región cervical, se utiliza el término disfunción craneocervicomandibular (DCCM). Esta condición puede contribuir para alteraciones coclear y vestibular, donde la primera se justifica por el origen embrionario y la proximidad anatómica de la ATM al oído y la segunda, por la disfunción de los músculos masticatorio y cervical (masetero y esternocleidomastoideo) que, debido a que son antigravitacionales, contribuyen a la vestibulopatía y también a las otalgias reflejas. A pesar de estas relaciones existentes, su patogénesis no se comprende completamente. El objetivo de este trabajo es presentar un caso clínico de diagnóstico y tratamiento de paciente con DCCM asociado con alteración vestibulococlear.

Descriptores: Articulación Temporomandibular; Dolor de Cuello; Puntos Disparadores; Trastornos Craneomandibulares; Síndromes del Dolor Miofascial.

INTRODUÇÃO

Okeson $^{1}$ definiu o sistema estomatognático como uma unidade funcional do corpo altamente refinada e complexa, em que tecidos diferentes, quanto à origem e estrutura, agem harmoniosamente na realização de diversas tarefas funcionais. $O$ sistema é composto por ossos, ligamentos, músculos e a articulação temporomandibular (ATM). A mastigação, a deglutição, a fala e a postura dependem muito da saúde e da estabilidade dessa articulação.

Ordinariamente, alterações estruturais e funcionais do sistema estomatognático podem dar origem à Disfunção Temporomandibular (DTM) e, quando associadas a comprometimento da musculatura cervical de contiguidade, denomina-se disfunção craniocervicomandibular (DCCM $)^{2,3}$. A causa desta disfunção está relacionada a diversos fatores ascendentes ou descendentes, utilizando como perspectiva o sistema mastigatório: traumas, alterações oclusais e posturais, condições ocupacionais, doenças sistêmicas, transtornos internos do disco, hipo ou hipermobilidade articular, disfunções miofasciais e/ou vertebrais cervicais, desgastes ósseos, estresse emocional, entre outros ${ }^{4}$.
Pode-se citar como os principais sinais e sintomas das DTMs: cefaleias; otalgias; odontalgias; zumbidos; plenitude auricular; dor miofascial, esta geralmente detectada por meio de palpação na musculatura mastigatória e cervical; dor articular; limitações no movimento mandibular por conta de desarranjos do disco articular sem redução; e estalidos/ruídos nas ATMs quando há redução ${ }^{5,6}$.

Neville et al. ${ }^{7}$, citam que a dor normalmente se localiza na área pré-auricular irradiando para a região temporal, frontal ou occipital; e os pontos gatilhos (PGs) miofasciais, que são caracterizados por regiões circunscritas hipersensíveis no interior dos músculos, são os grandes responsáveis pela sintomatologia dolorosa local ou referida sendo uma fonte constante de dor.

Normalmente os PGs são identificados por meio de palpação e podem ser classificados em ativos e latentes. O ponto gatilho (PG) ativo é caracterizado por apresentar dor espontânea ou surge ao movimento da musculatura mastigatória e/ou cervical. É um foco de hiperirritabilidade, localizado em musculatura tensa em áreas que geralmente o 
paciente relata dor. Em contrapartida, os PGs latentes estão presentes em áreas assintomáticas, não são associados à dor espontânea, mas geram dor ao serem estimulados à palpação, podendo se tornar ativos diante de agentes exógenos ou endógenos, bem como os ativos podem se tornar latentes ${ }^{8,9}$.

A localização do PG também pode ser obtida em exames de sonoelastografia (elastografia associada ao ultrassom), elastografia por ressonância magnética (ERM) e termografia infravermelha (TIN) ${ }^{10-12 .}$ A elastografia é um exame de imagem que mapeia propriedades elásticas e rigidez dos tecidos moles, sendo útil para avaliar a gravidade das condições que alteram a consistência dos tecidos.

A relação anatômica e neurofuncional entre o crânio, ATM e a coluna cervical justifica a frequente associação entre DTM e dores nos músculos mastigatórios e cervicais ${ }^{2}$. Uma correlação entre DTM e postura corporal foi possível após análises das complexas interações anatômicas e biomecânicas, em que a atividade aumentada da musculatura mastigatória leva a anteriorização cérvico-escapular, através da interferência nos músculos de contra-apoio, esternocleidomastoideo e trapézio, o que faz com que a projeção anteriorizada do corpo acarrete em tensão, e posteriormente, em disfunção na musculatura que pode levar à alterações vestibulares que concorrem para vestibulopatias e também otalgias reflexas ${ }^{13,14}$.

Em contrapartida, as alterações cocleares são justificadas pela origem embrionária da mandíbula e dos ossículos da orelha média a partir da cartilagem de Meckel, destarte, anatomicamente, a ATM estar muito próxima da orelha, assim como suas artérias, veias e nervos serem inter-relacionados, onde qualquer alteração de sua fisiologia, tem potencial de gerar prejuízo a estas estruturas adjacentes ${ }^{6,15}$. Outro fator muito importante diz respeito às vias sensitivas que transmitem estímulos até áreas cerebrais responsáveis pela sua percepção, em que o nervo trigêmeo surge como o elemento mais importante no aspecto da sensibilidade craniofacial ${ }^{3,16}$.

Neste contexto, a dor referida é explicada por Okeson ${ }^{1}$ de duas maneiras; na primeira diz que por conta de estímulos constantes e prolongados em um interneurônio, há um acúmulo de substância neurotransmissora na sinapse, a qual pode atingir outro interneurônio circunvizinho, tornando-o também excitado. $\mathrm{O}$ cérebro percebe a nocicepção sendo transmitida pelos dois neurônios, com isso, a percepção do estÍlmulo doloroso pelo cérebro a partir deste segundo neurônio é denominado dor heterotópica (dor referida). Outra explicação diz sobre a convergência neuronal, em que muitos neurônios fazem sinapse com um único interneurônio que converge com um próximo interneurônio ascendente e com a aproximação ao tronco encefálico e ao cortéx, a localização precisa do estímulo se torna cada vez mais difícil para o córtex por conta da presença de dor profunda e contínua, resultando na percepção de dor em estruturas normais (dor heterotrópica).

Outras alterações podem estar associadas à DCCM, o que pode ser um enorme desafio para os profissionais da saúde, tanto no fechamento do diagnóstico quanto no manuseio clínico ${ }^{17,18}$.

Um questionário anamnésico elaborado por Fonseca et al. $^{19}$ pode ser utilizado como um importante instrumento para caracterizar e classificar os pacientes com DTM em categorias de severidade dos sintomas em leve, moderada e grave.

Muitas alternativas de tratamentos existem para pacientes com DTM, podem ser citadas algumas como: psicoterapia, terapia medicamentosa, placas miorrelaxantes, exercícios fisioterápicos e, em alguns casos, até mesmo a cirurgia de ATM, valendo ser ressaltado que o cirurgião-dentista, para nortear o tratamento mais indicado para cada caso, deve avaliar de forma criteriosa e individualizada ${ }^{20}$

Desta maneira, investigações direcionadas aos fatores etiológicos que visam identifica-los e controla-los, principalmente em seus estágios iniciais de desenvolvimento, são de extrema importância para alcançar um correto diagnóstico, e posteriormente, para que as abordagens terapêuticas tenham o sucesso esperado, muitas vezes estas etapas clínicas podendo envolver uma equipe multidisciplinar ${ }^{3,21}$.

\section{CASO CLÍNICO}

Paciente de sexo feminino, leucoderma, 55 anos, procurou a clínica de Estomatologia da Policlínica Odontológica da UEA, queixando-se de dores que ascendiam da região cervical à temporal bilateralmente. Inicialmente, no exame extraoral foi observado um encurtamento do lado direto em relação ao esquerdo (Figura 1). À palpação, foi detectada presença de pontos gatilhos nos músculos cervicais e mastigatórios: trapézio descendente, esternocleidomastóideo, masseter e temporal, bilateralmente, porém com predominância dolorosa e de reação de fuga à palpação no lado direito (Figura 2). Foi relatada a presença de zumbido e plenitude auricular acentuada no lado direito, recorrentes dores de cabeça, além de tontura (vertigem), e que há sete meses enquanto realizava suas atividades laborais começaram os episódios dolorosos nas regiões cervical e facial. Foi mensurada a abertura máxima de boca (Figura 3), assim como a avaliação de restrição mandibular excêntrica (Figura 4 e 5). Os exames foram refeitos três meses após o início do tratamento e pôde-se observar melhora clínica significativa, tendo em vista os valores obtidos na reavaliação. Foi observada interferência mandibular com deflexão a partir de 5 milímetros de abertura, em protrusão, não ocorrendo desvio. 


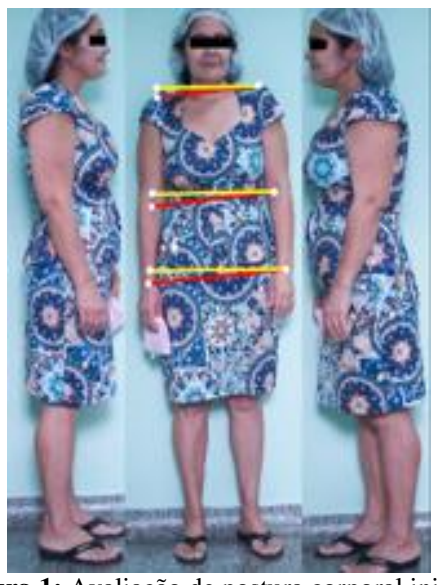

Figura 1: Avaliação de postura corporal inicial.

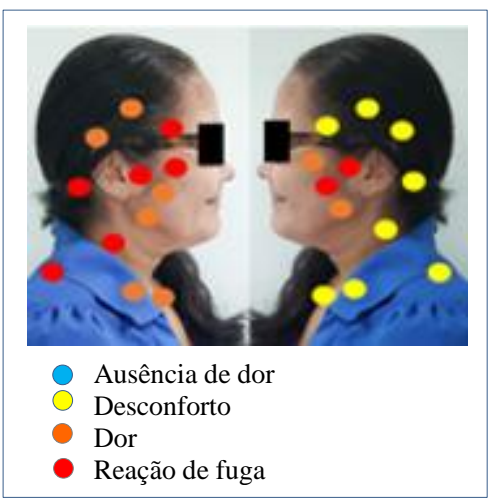

Figura 2: Avaliação inicial de pontos gatilhos.

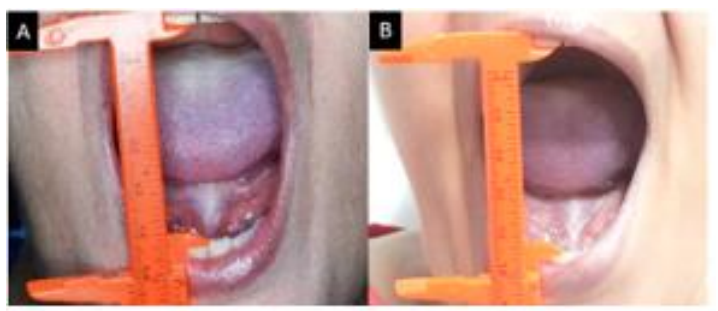

Figura 3: Mensuração de abertura bucal máxima. A, mensuração de abertura de quarenta e cinco milímetros inicialmente. B, mensuração de abertura de quarenta e seis milímetros após três meses de tratamento.

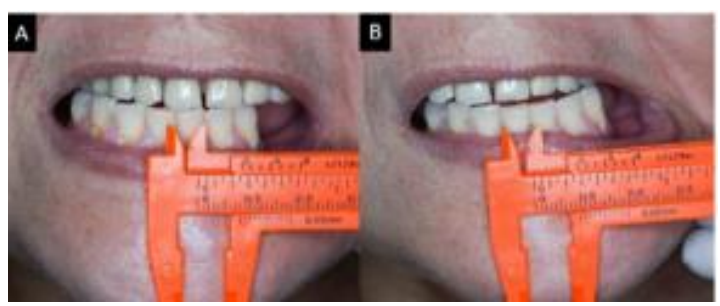

Figura 4: Restrição mandibular excêntrica no exame inicial. A, restrição mandibular excêntrica direita de dois milímetros. B, restrição mandibular excêntrica esquerda de três milímetros.

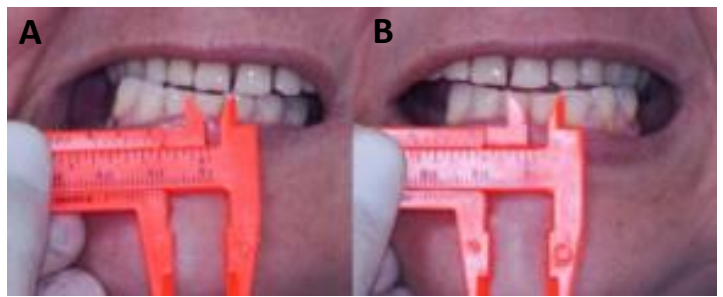

Figura 5: Restrição mandibular excêntrica após três meses. A, restrição mandibular excêntrica direita de cinco milímetros. $\mathrm{B}$, restrição mandibular excêntrica esquerda de cinco milímetros.

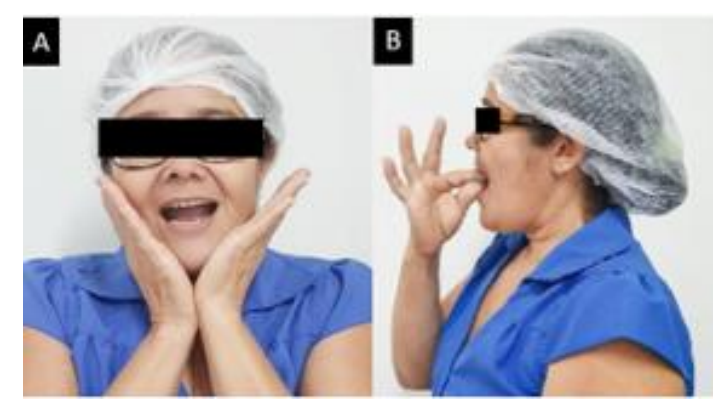

Figura 6: Exercícios fisioterápicos. A, exercício fisioterápico de abertura bucal contra resistência. B, exercício fisioterápico de abertura e fechamento bucal orientado.

Não foram relatados episódios de luxação, nem presença de estalido ou crepitação nas ATMs, mas foi informado o hábito parafuncional de ranger os dentes. A partir dos achados clínicos chegou-se ao diagnóstico de disfunção miofascial craniocervicomandibular (DCCM) ascendente associada à alteração vestibulococlear. O tratamento inicial consistiu-se em infiltração com lidocaína $2 \%$ em pontos gatilhos em músculo trapézio descendente direito e, posteriormente, a paciente foi orientada a realizar exercícios fisioterápicos (Figura 6), como abertura bucal contra resistência e abertura e fechamento orientados associados ao uso de placa interoclusal, 3 vezes ao dia com duração de uma a duas horas (Figuras 7 e 8).

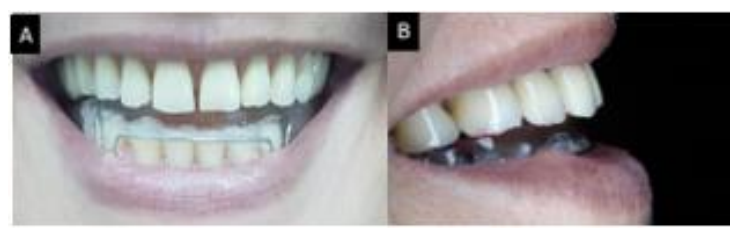

Figura 7: Adaptação e ajuste oclusal da placa interoclusal. A, vista frontal. B, vista lateral.

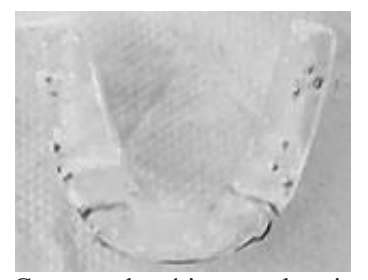

Figura 8: Contatos dentários em placa interoclusal.

Para a confecção da placa miorrelaxante foi realizado a moldagem das arcadas superior e inferior da paciente para obtenção dos modelos de estudos (Figura 9), bem como o registro do arco facial (Figura 10) para então, encaminhá-los ao laboratório protético.

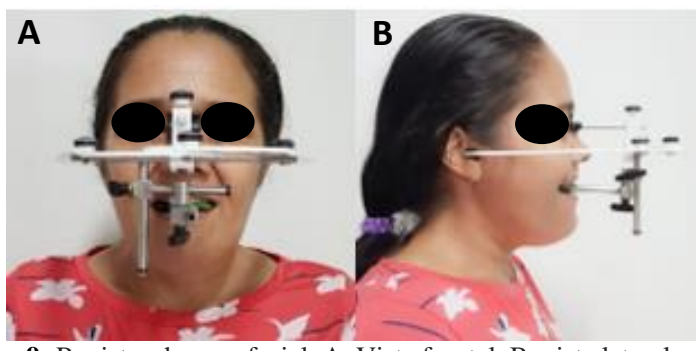

Figura 9: Registro do arco facial. A, Vista frontal. B, vista lateral. 


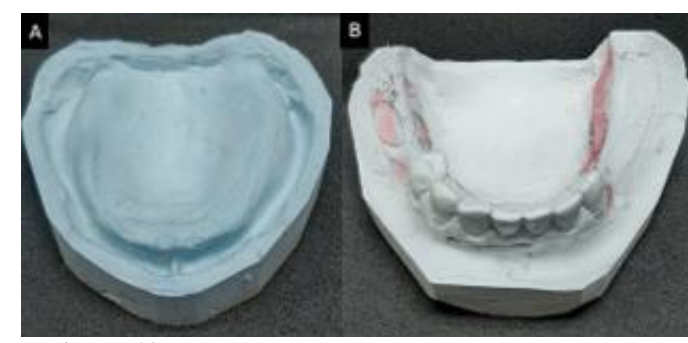

Figura 10: Modelos de estudo, A. Superior. B, inferior.

Após três meses de tratamento observou-se melhora significativa do quadro de acordo com a avaliação clínica e percepção subjetiva da paciente, não ocorrendo reação de fuga, nem dor, quando submetida à palpação nas regiões maxilomandibulares e cervical bilaterais (Figura 11). Seguindo o plano de tratamento global será realizado a confecção da prótese total superior, tendo em vista o desgaste acentuado da prótese atual da paciente que consequentemente, faz com que haja uma diminuição da dimensão vertical, caracterizado por um encurtamento do terço inferior da face, e posteriormente, confecção de uma prótese parcial inferior.

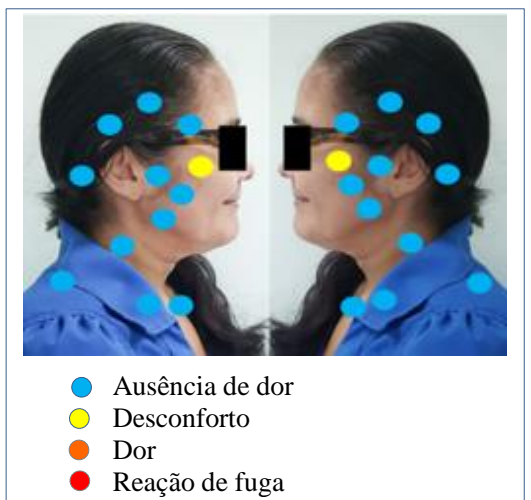

Figura 11: Reavaliação de Pontos Gatilhos após três meses do tratamento.

DISCUSSÃO

No presente estudo constatou-se a melhora significativa do quadro sintomático e dos sinais de disfunção da paciente a partir do tratamento clínico implementado, em acordo com metodologias descritas por um amplo conjunto de autores.

A partir do índice anamnésico foi possível obter um correto diagnóstico e posteriormente, traçar um plano de tratamento para o caso. Os dados coletados na anamnese mostraram relato de dor que variava quanto sua intensidade, ao exame físico, desde desconforto até o extremo, observada com reação de fuga à palpação dos PGs, associada a outros achados clínicos vestibulococleares. Estes dados corroboram com a informação de que a dor é o sintoma mais significativo em DTMs e molesta quase $20 \%$ da população ocidental ${ }^{22-24}$.

Estas condições podem ocorrer simultaneamente com as disfunções cervicais, originando-as por descendência de contiguidade, por serem os músculos do pescoço contra-apoio dos músculos mastigatórios ou estes serem comprometidos ascendentemente, caracterizando um quadro que envolve este sistema e que faz parte de um dos 3 pontos de equilíbrio postural denominado manducatório, manifestando em ambos os casos a condição como uma $\mathrm{DCCM}^{22-24}$.

Sinais e sintomas nas ATMs como cliques, rangidos ou crepitações podem vir acompanhados de limitações dos movimentos mandibulares e cervicais, e os sintomas otoneurológicos: zumbido, plenitude auricular e vertigem/tontura podem também estar presentes ${ }^{6,25}$.

Esta afirmação converge para o que foi observado no caso clínico, em que a paciente relatava tontura, limitação nos movimentos de abertura/fechamento bucal e na região cervical; havia também presença de zumbido e plenitude auricular acentuado na orelha direita, no entanto não havia presença de estalido, nem crepitação nas ATMs.

Vale ser ressaltado que esta ausência de estalidos e crepitações nas ATMs não é necessariamente sinônimo de uma articulação saudável, uma vez que o deslocamento do disco articular sem redução pode não provocar ruído $\operatorname{articular}^{1,25}$.

À palpação foi detectado que os músculos mais dolorosos, com reação de fuga, foram masseter, temporal e esternocleidomastoideo, com exacerbação da sintomatologia no lado direito da face. Porém, na literatura ainda não há concordância a respeito da região muscular mais acometida pela dor, todos os músculos orofaciais podem apresentar um quadro álgico em função de parafunções, hipermobilidade muscular, entre outras causas ${ }^{26-28}$.

Assim, a identificação de quais músculos respondem com sintomatologia à palpação de PGs é importante para explicar os sintomas auditivos, como observaram Jacob et al. ${ }^{29}$. Neste estudo, os sintomas otológicos (dor referida na orelha, plenitude auricular e tontura) nos pacientes com DTM estavam presentes de forma significativa e correlacionados à palpação positiva dos músculos esternocleidomastoideo e masseter, que por serem músculos antigravitacionais relacionados à postura podem também concorrer para as alterações vestibulares.

As vestibulopatias podem ser, primariamente, resultado da atividade aumentada da musculatura mastigatória que leva, de forma descendente, à anteriorização cervicoescapular, através de interferência nos músculos de contra-apoio, esternocleidomastoideo e trapézio, o que pode comprometer o fornecimento de estímulos sensoriais ao SNC sobre a posição da cabeça ou ascendentemente, advindos da musculatura cervical com comprometimento secundário da musculatura mastigatória, acarretando em tontura (vertigem), desequilíbrio, falta de coordenação de movimentos, bem como otalgias reflexas ${ }^{13,14}$.

Outra explicação, para as alterações 
otológicas, seria o músculo tensor do véu palatino sofrer alteração quanto a sua atuação na abertura da tuba auditiva por conta de dissipação de espasmos advindos dos músculos mastigatórios ocasionando pressão negativa na orelha, otalgia, zumbido, cefaleia e perda da audição ${ }^{6,30}$.

O tratamento adequado é outro fator importantíssimo para alcançar resultados satisfatórios, com isso, a instituição do programa terapêutico deve obedecer a um modelo interdisciplinar, pois o controle da dor e da incapacidade física implica a necessidade de reabilitação física, psíquica e social ${ }^{4}$.

Com isso, como medida terapêutica inicial foi realizada a infiltração de lidocaína no pontogatilho do músculo trapézio descendente do lado direito com a finalidade de inativação do PG e alívio da dor referida. Após este alívio, recomendou-se a associação de exercícios fisioterápicos com o uso de placa miorrelaxante, uma vez que o tratamento inicial deve ser reversível e não invasivo ${ }^{1}$.

A fisioterapia tem um papel importante no tratamento das desordens temporomandibulares, objetivando o alívio da dor musculoesquelética, redução da inflamação e restauração da função motora normal. Dentre as intervenções adotadas, citam-se as modalidades de exercícios e técnicas de terapia manual. Os exercícios priorizam a musculatura mastigatória e da região cervical para a melhora da força e mobilidade da região, já a terapia manual é usada geralmente para melhora da dor e restauração da mobilidade articular. Os exercícios utilizados no caso clínico foram: abertura bucal contra resistência, fechamento bucal orientado e reorganização postural com auxílio de bastão ${ }^{28}$.

Quanto ao uso do dispositivo interoclusal, uma das suas utilidades é a reorganização proprioceptiva da musculatura mastigatória e cervical permitindo, por conseguinte, que a ATM adote uma posição musculoesquelética (ME) mais estável, que é a relação cêntrica $(\mathrm{RC})$, por fornecer uma condição oclusal o mais próxima do ideal. Esta reorientação espacial proporciona uma reorganização da atividade neuromuscular reflexa, reduzindo a atividade muscular anormal enquanto estimula a função muscular normal ${ }^{1}$.

Contudo, a efetividade das placas interoclusais têm-se mostrado baixa quando utilizada como única modalidade de tratamento, devendo atuar como parte da terapêutica, ou até mesmo como coadjuvante de outras terapias, como a medicamentosa ou a fisioterápica, sendo necessário que se descubra a etiologia da disfunção miofascial mastigatória para que se obtenha sucesso a longo prazo no tratamento ${ }^{31}$.

$\mathrm{O}$ uso do dispositivo interoclusal miorrelaxante foi um adjuvante importante quando associado a outras terapias, para a resolução da queixa apresentada: infiltração de lidocaína em pontos gatilhos de músculo de contra-apoio relacionado e exercícios fisioterápicos para reorganização postural e da cintura escapular, para reorganização do sistema manducatório e estabilização vestibular. O sinergismo da abordagem terapêutica concorreu para o resultado satisfatório.

CONCLUSÃO

Tendo em vista a complexidade e o caráter multifatorial da DCCM, é indispensável que o profissional saiba identificar a etiofisiopatogenia da condição e, a partir de então, lançar mão de uma combinação de abordagens terapêuticas, sendo estas mais eficazes do que terapias isoladas. É necessário, portanto, a valorização de todos esses aspectos propedêuticos e a construção de uma relação de parceria e corresponsabilidade com o paciente disfuncional durante a execução do programa de tratamento e de reabilitação, para que haja sucesso terapêutico.

\section{REFERÊNCIAS}

1. Okeson JP. Tratamento das desordens Temporomandibulares e Oclusão. 6.ed. Rio de Janeiro: Elsevier; 2008.

2. Pozzebon D, Piccin CF, Silva AMT da, Corrêa ECR. Disfunção temporomandibular e dor craniocervical em profissionais da área da enfermagem sob estresse no trabalho. Rev CEFAC. 2016;18(2):439-48.

3. Godinho GV, Cabral LN. Disfunção craniocervicomandibular e alterações vestibulococleares: revisão de literatura. Arch Health Invest. 2019;8(8):405-12.

4. Mourão NLA, Mesquita VT. A importância da fisioterapia no tratamento das disfunções da ATM. Ter Man. 2006;4(16):66-9.

5. Santos PPA, Santos PRA, Souza LB. Características gerais da disfunção temporomandibular: conceitos atuais. Rev nav odontol. 2009;3(1):10-3.

6. Soares PG, Cabral LN. Disfunção temporomandibular associada à cocleopatia: relato de caso. Arch Health Invest. 2019;8(12):789-94.

7. Neville BW, Damm DD, Allen CM, Bouquet JE. Patologia Oral e Maxilofacial. $3^{\circ}$. Rio de Janeiro: Elsevier; 2009.

8. Yeng LT, Kaziyama HHS, Teixeira MJ. Síndrome Dolorosa Miofascial. JBA Curitiba. 2003; 3(9):27-43.

9. Gal P, Kaziama H, Lin T, Teixeira M, Correa C. Síndrome miofascial. Abordagem fisiátrica. Arq Bras Neurocirurg. 1991;10(4):181-87.

10. Skorupska E, Rychlik M, Samborski W. Validation and test-retest reliability of new thermographic technique called thermovison technique of dry needling for gluteus minimums trigger points in sciatica subjects and TrPs- 
negative healthy volunteers. Biomed Res Int. 2015;2015:546497.

11. Haddad DS, Brioschi ML, Arita ES. Thermographic and clinical correlation of myofascial trigger points in the masticatory muscles. Dentomaxillofac Radiol. 2012;41(8):621-29.

12. Unverzagt C, Berglund K, Thomas JJ. Dry needling for myofascial trigger point pain: a clinical commentary. Int J Sports Phys Ther. 2015;10(3):402-18.

13. Amantéa DV, Novaes AP, Campolongo GD, Barros TP. A importância da avaliação postural no paciente com disfunção da articulação temporomandibular. Acta ortop bras. 2004;12(3):155-59.

14. Robocado SM. Cabeza y Cuello. Tratamento Articular. Inter Médica Editor. 1979.

15. Farias KR. Sinais e sintomas de desordens temporomandibulares em pacientes portadores de disfunção vestibular [monografia]. Campina Grande: Universidade Estadual da Paraíba; 2015.

16. Rabello GD. Dores orofaciais. In: Manganello LC, Silveira ME, Silva AA. Cirurgia de articulação temporomandibular. São Paulo: Gen; 2014.

17. Campi LB, Jordani PC, Tenan HL, Camparis CM, Gonçalves DA. Painful temporomandibular disorders and central sensitization: implications for management-a pilot study. Int $\mathrm{J}$ Oral Maxillofac Surg. 2017;46(1):104-10.

18. Costa YM, Conti PC, de Faria FA, Bonjardim LR. Temporomandibular disorders and painful comorbidities: clinical association and underlying mechanisms. Oral Surg Oral Med Oral Pathol Oral Radiol. 2017;123(3):288-97.

19. Fonseca DM, Bonfate G, Valle AL, Freitas SFT. Diagnóstico pela anamnese da disfunção craniomandibular. Rev Gaucha Odontol. 1994;42(1):23-8.

20. Schiffman EL, Fricton JR, Haley DP, Shapiro BL. The prevalence and treatment needs of subjects with temporomandibular disorders. J Am Dent Assoc. 1990;120(3):295-303.

21. Maydana AV, Tesch RS, Denardin OVP, Ursi WJS, Dworkin SF. Possíveis fatores etiológicos para desordens temporomandibulares de origem articular com implicações para diagnóstico e tratamento. Dental Press J Orthod. 2010; 15(3):78-86.

22. Pessoa CP, Barreto MB, Santos LB, Alves TDB, Oliveira MC, Martins AG. Instrumentos utilizados na avaliação do impacto da dor na qualidade de vida de pacientes com dor orofacial e disfunção temporomandibular. Rev Baiana Saúde Pública. 2007;31(2):267-93.

23. Nomura K, Vitti M, Oliveira AS, Chaves TC, Semprini M, Siéssere S, Hallak JE, Regalo SC.
Use of the Fonseca's questionnaire to assess the prevalence and severity of temporomandibular disorders in Brazilian dental undergraduates. Braz Dent J. 2007;18(2):163-7.

24. Oliveira AS, Bevilaqua-Grossi D, Dias EM. Sinais e sintomas da disfunção temporomandibular nas diferentes regiões brasileiras. Fisioter Pesqui. 2008;15(4):392-96.

25. American Society of Temporomandibular Joint Surgeons. Guidelines for diagnosis and management of disorders involving the temporomandibular joint and related musculoskeletal structures. Cranio. 2003;21(1):68-76.

26. Kinote APB de M, Melo LTM, Vieira AAC, Ferreira NMN, Abdon AP de V. Perfil funcional de pacientes com disfunção temporomandibular em tratamento fisioterápico. Rev Bras Promoç Saúde. 2011;24(4):306-12.

27. Gonçalves RN, Ordenes IEU, Bigaton DR. Efeito indireto da TENS sobre os músculos cervicais em portadores de DTM. Fisioter Mov. 2007; 20(2):83-90.

28. Oliveira KB, Pinheiro ICO, Freitas DG, Gualberto HD, Carvalho NAA. A abordagem fisioterapêutica na disfunção da articulação temporomandibular. Revisão da literatura. Med Reabil. 2010;29(3):61-4.

29. Jacob LCB, Rabiço TM, Campêlo RM, Aguiar FP, Zeigelboim BS. Sintomas auditivos e análise das emissões otoacusticas evocadas por estímulo transiente em indivíduos portadores de disfunção temporomandibular. Rev Disturb comun. 2005;17(2):173-82.

30. Arlen $\mathrm{H}$. The otomandibular syndrome. In: Gelb $\mathrm{H}$. Clinical management of head, neck and TMJ pain and dysfunction. Philadelphia: Sauders; 1985.

31. Portero PP, Kern R, Kusma SZ, Grau-Grullón P. Placas oclusais no tratamento da disfunção temporomandibular (DTM). Rev Gestão \& Saúde. 2009;1(1):36-40. 


\section{CONFLITO DE INTERESSES}

Os autores declaram não haver conflitos de interesse.

\section{AUTOR PARA CORRESPONDÊNCIA}

\section{Inácio Bruno Romano Ribeiro}

Rua São Jorge, n³, Bairro Colônia Santo Antônio

69093270. Manaus - AM, Brasil

E-mail: inacio.bruno11@gmail.com

Submetido em 19/07/2020

Aceito em 22/11/2020 